\title{
ÍNDICE DE PRODUTIVIDADE NA INDÚSTRIA DE LATICÍNIOS: PREVISÃO COM BASE EM MODELAGEM DE SÉRIES TEMPORAIS
}

\author{
Deise Graziele Dickel \\ Universidade Federal de Santa Maria \\ deisedickelsm@yahoo.com.br \\ Adriano Mendonça Souza \\ Universidade Federal de Santa Maria \\ amsouza.sm@gmail.com \\ Julio Cezar Mairesse Siluk \\ Universidade Federal de Santa Maria \\ jsiluk@ufsm.br \\ Simone Caberte Naimer \\ Universidade Federal de Santa Maria \\ simone.naimer@live.com
}

\begin{abstract}
Resumo
O índice de produtividade é um dos indicadores mais utilizados para a mensuração da competitividade na indústria, desta forma, avaliar este índice e, principalmente, poder prever a produtividade que uma indústria alcançará no futuro próximo é de fundamental relevância ao gestor a fim de que este consiga visualizar a performance da mesma frente ao mercado. Consonante a isto, a pesquisa objetivou realizar previsões do índice de produtividade no curto prazo em uma indústria de laticínios, assim como determinar o processo gerador da série analisada. Para isto, foi utilizada a metodologia de Box e Jenkins de modelagem para os Autoregressive Integrated Moving Average Models (ARIMA) em um conjunto de dados do índice de produtividade mensal do período compreendido entre 2009 e 2013 . Como principais resultados obteve-se a identificação de um modelo de médias móveis de ordem 1, MA(1), com uma defasagem no período 3 , denotando que as possíveis falhas no processo repercutem na produção por três meses.
\end{abstract}

Palavras-chave: Séries Temporais, Econometria, Performance Industrial, Competitividade.

\begin{abstract}
The productivity index is one of the most frequently used indicators to measure competitiveness in the industry thus review this index and especially to predict the productivity that an industry will achieve in the near future is of fundamental relevance to the manager so that this can view the performance thereof opposite the market. Consonant with this, the research aimed to realize productivity index forecasts in the short term in a dairy industry, as well as determine the generating process of the study period. For this, we used the Box Jenkins methodology and modeling for Autoregressive Integrated Moving Average Models (ARIMA) in a data set of monthly productivity index for the period between 2009 and 2013. The main results obtained the identification one moving average model of order 1, MA (1), with a lag in period 3, indicating that the possible flaws in the process impacting on production for three months.
\end{abstract}

Keywords: Time Series, Econometrics, Industrial Performance, Competitiveness. 


\section{INTRODUÇÃ̃O}

As indústrias têm passado por períodos de intensa competitividade em que os processos e tecnologia se mostram cada vez mais eficazes e qualificados. Com isso, fica cada vez mais evidente a busca por ferramentas que auxiliem no melhoramento do desempenho de suas atividades. As indústrias internacionais ganham mercado aprimorando os meios de produção e investindo em pesquisa. Assim, para que as indústrias brasileiras não percam espaço, é necessário que estejam atentas ás mudanças, bem como tenham subsídios completos para tomada de decisão (DRUCKER, 2008 ${ }^{[1]}$; PORTER, 2009 ${ }^{[2]}$; TEMPLAR, 2011 ${ }^{[3]}$; IRELAND, HOSKISSON e HITT, 2014 ${ }^{[4]}$ ).

A competitividade como eficiência é definida pela habilidade da organização em fabricar produtos e serviços melhores do que seus concorrentes, de acordo com os limites impostos pela sua capacitação humana, tecnológica, gerencial, financeira e comercial. Traduzida nas relações insumo-produto ou custo-preço, a competitividade é delimitada pelo produtor, e mensurável por indicadores como produtividade e técnicas de produção, em comparação àqueles apresentados pelas organizações de maior rendimento do setor (MACHADO DA SILVA e FONSECA, 2010 ${ }^{[5]}$; PAIS, GOMES e CORONEL, 2012 $2^{[6]}$; NEUENFELDT JÚNIOR, SILUK e SILVEIRA, 2013 ${ }^{[7]}$ ).

Para Porter (2009) ${ }^{[2]}$, o único conceito significativo de competitividade em nível nacional é a produtividade. O autor sugere que se deve abandonar toda a ideia de nação competitiva como expressão que tenha grande significado para a prosperidade econômica. A produtividade das empresas é o que realmente influencia e determina os níveis da prosperidade econômica de um país. Por outro lado, ele afirma que nenhuma nação pode ser competitiva em tudo. Para encontrar as respostas e diagnósticos, deve-se focalizar não a economia como um todo, mas em indústrias específicas e segmentos da indústria (MANÃS e PACANHAN, 2004 ${ }^{[8]}$; ZAGO, RIGONI, ABREU e RODRIGUEZ, 2008 ${ }^{[9]}$; FONTENELE, $2010^{[10]}$; MOREIRA, MOUTINHO e PEREIRA, 2013 ${ }^{[11]}$ ).

Dessa maneira, a mensuração do desempenho pode ser considerada como um parâmetro básico de comparação organizacional, sendo bastante aceita a premissa pela não utilização de avaliações baseados somente em abordagens subjetivas ou empíricas, e sim tomando por consideração os fatores devidamente contextualizados, havendo para tanto um método que seja capaz de informar mais do que se sabia antes a respeito do sistema (CARLINI JUNIOR e VITAL, 2004 ${ }^{[12]}$; NEELY, 2005 ${ }^{[13]}$; BITITCI, MENDIBIL, MARTINEZ e ALBORES, 2005 ${ }^{[14]}$; SLACK, SLACK, CHAMBERS e JOHNSTON,

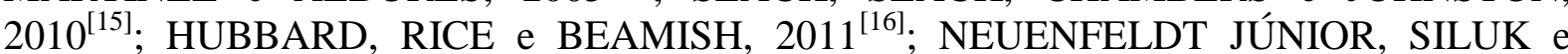
SILVEIRA, $2013^{[7]}$.

A competitividade pode ser determinada por vários indicadores específicos de cada setor, sendo o índice de produtividade um dos mais importantes para a indústria e, citando o caso da indústria de alimentos, este índice pode ser determinante para a sobrevivência de uma empresa. Além disso, o setor alimentício tem se mostrado determinante também para o desenvolvimento do país. Segundo a Associação Brasileira das Indústrias da Alimentação $(\mathrm{ABIA})^{[17]}$, em 2012 o setor atingiu faturamento de cerca de $\mathrm{R} \$ 431,9$ bilhões, além de empregar cerca de 1,3 milhão de pessoas.

Dentro do contexto de alimentação, destaca-se o setor de produtos derivados do leite, pois, de acordo com dados do IBGE (2011) ${ }^{[18]}$, e Milkpoint (2012) ${ }^{[19]}$, o mercado brasileiro é um dos seis maiores do mundo, com destaque especial para o Rio Grande do Sul, que totaliza $12,1 \%$ da produção nacional.

Contudo, conforme Lírio, Souza e Da Silva $(2003)^{[20]}$, a atividade do ramo de produção de leite é caracterizada como um negócio de lucros reduzidos, o que impõe que 
somente permanecerão no mercado aqueles que consigam reduzir os seus custos e aumentar o volume de produto a ser comercializado. De acordo com Porter (2009) ${ }^{[2]}$, a tecnologia e a informação tornaram-se fatores essenciais e fundamentais para as empresas suportarem a dinâmica do mercado em meio a mudanças rápidas de uma economia globalizada, sendo importante para a implementação da estratégia competitiva e a busca de inovações.

Dentro deste contexto, o método econométrico de previsão por meio de séries temporais pode ser um grande aliado na modelagem de previsão dos índices de produtividades destas empresas. Este método tem sido largamente utilizado para previsão de demanda e também para previsão de erros de produção, contudo, poucos trabalhos tem abordado a previsão do índice de produtividade, o que pode ser considerado um diferencial para a pesquisa, auxiliando os gestores a programarem suas ações estratégicas de acordo com os resultados destas previsões.

A análise de séries temporais tem importante papel na previsão de cenários no mundo empresarial, sendo definidas por Gujarati $(2000)^{[21]}$, como uma metodologia que compreende a identificação da estrutura de uma série temporal, bem como a criação de um modelo que permita realizar projeções. Pode-se definir uma série temporal como um conjunto de observações de uma dada variável, ordenadas no tempo e colhidas em intervalos iguais. Os modelos de séries temporais possibilitam descrever um processo estocástico utilizando apenas valores passados da variável dependente e do termo de erro (ANDRADE, 2011 ${ }^{[22]}$ ).

Os Autoregressive Integrated Moving Average Models (modelos ARIMA) baseiam-se na teoria de que o comportamento da própria variável pode responder por suas dinâmicas futuras (BOX e JENKINS, 1970 ${ }^{[23]}$ ). A estimativa de parâmetros é geralmente realizada utilizando o método de mínimos quadrados ordinários ou máxima verossimilhança e testes de diagnóstico residuais são realizados para validar o modelo e realizar a previsão (SOUZA, SOUZA, FERREIRA e MENEZES, $2011^{[24]}$ ).

Assim, a presente pesquisa tem como objetivo realizar previsões do índice de produtividade no curto prazo em uma indústria de laticínios, assim como determinar o processo estatístico gerador da série analisada, como forma de buscar subsídios para que a mesma se torne mais competitiva no mercado nacional e internacional, podendo corrigir falhas no seu processo a fim de prever o comportamento do seu índice de produtividade.

A pesquisa justifica-se dada a relevância do setor de laticínios na cadeia produtiva nacional, as possíveis crises com diminuições ou paradas da produção e os prejuízos causados, que podem abranger toda a cadeia do setor econômico, pois as empresas não poderão manter o padrão de fornecimento nas distribuidoras, e assim não poderão atender à demanda da população (PORTER, 2009 ${ }^{[2]}$; STADLHOFER, 2010 ${ }^{[25]}$ ).

Para garantir esta disponibilidade ao consumidor dentro dos prazos estabelecidos, as decisões tomadas perante aos problemas produtivos necessitam ser rápidas, eficazes e balizadas em informações consistentes (JOSHI, NEPAL, RATHORE e SHARMA, 2013 ${ }^{[26]}$ ).

A organização escolhida para aplicação do método de previsão proposto é referência em produção de leite em pó em toda a América Latina, chegando a processar mais de 1 milhão de litros de leite por dia e exportando cerca de $50 \%$ da sua produção mensal. Em busca de maior eficiência nos seus processos, a organização em questão vem sofrendo um processo de adequação à cinco normas internacionais, sendo que seu sistema de gestão está baseado no atendimento dos requisitos de tais normas, publicadas por órgãos de acreditação internacional como o International Organization for Standardization (ISO) e o Occupational Health and Safety Assessment Series (OHSAS), que buscam a melhoria dos processos da indústria, bem como maior produtividade. 


\section{METODOLOGIA}

O estudo contemplou os registros do índice de produtividade coletados pelo pesquisador na própria indústria, com observações mensais durante cinco anos, no período compreendido entre janeiro de 2009 e dezembro de 2013, perfazendo um total de 60 observações. Os valores do índice de produtividade foram levantados a partir do volume de leite fluido que chega para a produção e o volume de leite em pó que é produzido e expedido para comercialização, sendo que o índice resultante pode assim medir a produtividade da indústria considerando a eficácia do processo. Dessa forma, quanto maior for o índice, mais eficiência a indústria alcançou no mês e quanto menor for o índice alcançado, menor foi à eficiência do processo, conforme mostra a Equação (1),

$$
i p=\frac{v l p x 100}{v l r}
$$

onde ip é o índice de produtividade, $v l p$ é o volume de leite produzido e $v l r$ corresponde ao volume de leite recebido pela fábrica.

Para o atingimento dos objetivos propostos, faz-se necessária a compreensão dos principais processos geradores dos modelos ARIMA, sendo que os mesmos podem ser enquadrados em 3 grupos principais. Os modelos autorregressivos (AR), os modelos de médias móveis (MA) ou ainda os modelos autorregressivos de médias móveis (ARMA).

Os modelos autorregressivos (AR) foram criados com a ideia de que a presente observação da série $Z_{t}$ pode ser explicada como uma função das $p$ observações passadas, onde $p$ determina o número de passos entre as observações passadas e a previsão da próxima observação. A estrutura autorregressiva geral é expressa pela Equação (2),

$$
Z_{t}=\varphi_{1} Z_{t-1}+Z_{t-2}+\cdots+\varphi_{p} Z_{t-p}+a_{t}
$$

de modo que $\varphi_{i}$ são parâmetros da estrutura, $i=1, \ldots, p$ (ordem da estrutura) e $a_{t}$ é ruído branco com média zero e variância $\sigma_{\alpha}^{2}$.

Já os modelos de médias móveis (MA) são formados por uma combinação linear do ruído branco, $a_{t}$, ocorridos no período corrente e nos períodos passados. A estrutura de médias móveis geral é expressa na Equação (3),

$$
Z_{t}=a_{t}-\theta_{1} a_{t-1}-\theta_{2} a_{t-2} \ldots-\theta_{q} a_{t-q}
$$

em que $\theta_{i}$ são parâmetros da estrutura, $i=1, \ldots, q$ (a ordem da estrutura) e $a_{t}$ é ruído branco com média zero e variância $\alpha_{\alpha}^{2}$.

O modelo autorregressivo de médias móveis é uma combinação dos dois anteriores onde $Z_{t}$ é descrito por seus valores passados e pelos ruídos brancos correntes e passados. A estrutura geral ARMA $(p, q)$ é expressa na Equação (4),

$$
Z_{t}=\varphi_{1} Z_{t-1}+\varphi_{2} Z_{t-2}+\cdots+\varphi_{p} Z_{t-p}+a_{t}-\theta_{1} a_{t-1}-\theta_{2} a_{t-2}-\cdots-\theta_{q} a_{t-q}
$$

onde $\varphi_{i}$ são os parâmetros da estrutura autorregressiva, $i=1, \ldots, p, \theta_{i}$ são os parâmetros da estrutura médias móveis, $i=1, \ldots, q$ e $a_{t}$ é o ruído branco. 
As variáveis defasadas de $Z_{t}$ representam a parte autoregressiva do modelo (AR), enquanto que as defasagens do termo de erro representam a parte de média móvel $(M A)$, onde $p$ representa a ordem de defasagem do termo autoregressivo e $q$ a ordem de defasagem do termo de média móvel.

Para a identificação do processo gerador da série, foi utilizada a metodologia proposta por Box e Jenkins $(1970)^{[23]}$, por se mostrar a mais consagrada na área dos modelos ARIMA, cuja sistemática segue o demonstrado na Figura 1.

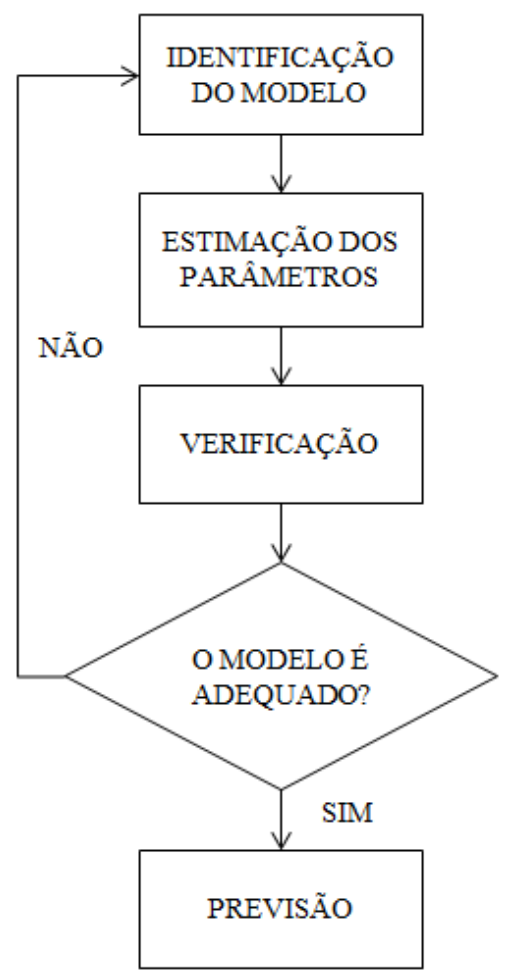

Figura 1 - Ciclo Interativo da metodologia. Fonte: Adaptado pelos autores.

A série original foi analisada sob a ótica das estatísticas descritivas para observação de possíveis indícios de discrepâncias. A seguir, os dados foram submetidos a avaliação das funções de autocorrelação (FAC) e autocorrelação parcial (FACP) com vistas à realização do primeiro diagnóstico visual. Após esta etapa foi necessário verificar a condição de estacionaridade da série, uma vez que para se aplicar a metodologia de Box e Jenkins ${ }^{[23]}$, a série em estudo tem que ser estacionária, ou seja, a série tem que ter média, variância e covariância finitas e constantes (MORETTIN e TOLOI, 2004 ${ }^{[27]}$ ).

Se a série em estudo for não estacionária, tem-se um ARIMA $(p, d, q)$, onde $\mathrm{d}$ representa a ordem de diferenciação. As diferenciações podem ser realizadas com a utilização da Equação (5).

$$
\Delta_{y}^{d}=y_{t}-y_{t-1}
$$

O exame da estacionaridade da série em questão foi realizado por meio do teste de raiz unitária Dickey-Fuller Aumentado $(\mathrm{DFA})^{[28]}$ e do teste de Kwiatkowski- Phillips-SchmidtShin (KPSS) ${ }^{[29]}$. 
Para a estimação dos parâmetros do modelo utilizou-se o software EViews $8 S V$ para encontrar os modelos concorrentes que explicassem o comportamento da série e permitissem a previsão, obtendo-se mais de um modelo concorrente, a utilização dos critérios Akaike Info Criterion (AIC) e do Schwarz Criterion (BIC) é aplicado para encontrar o modelo mais adequado. A verificação foi feita através dos gráficos de controle do ruído branco, sendo que todos os lags devem permanecer dentro dos mesmos. Por fim, a etapa de previsão ocorreu também por meio do software buscando uma previsão de curto prazo para o caso em questão.

\section{RESULTADOS}

Observando os dados relativos ao índice de produtividade na indústria de laticínios estudada, pode-se observar uma grande variabilidade dos dados por meio de gráfico de linha e das estatísticas descritivas da série original, cujo valor máximo do índice atingido foi 13,52 e o valor mínimo 3,86. Isto demonstra que a empresa ainda não conseguiu atingir uma estabilidade na produtividade, apresentando problemas durante o processo e discrepâncias muito grandes entre o volume de leite fluido que chega para a produção e o volume que é produzido.

Denota-se também que, grande parte do leite recebido é perdido durante as fases de análise inicial, em que são realizados ensaios laboratoriais para verificar a qualidade do leite, e também na fase de processamento por inúmeros motivos, podendo ser erros humanos, defeitos em equipamentos, falta de energia, dentre outros que afetam negativamente o processo.

A média do índice permaneceu em 10,39 e mediana de 11,06, mais próximas do número máximo, o que denota que os picos inferiores são minoria e confirmando que nestas quedas do índice podem ter havido problemas durante o processo. $\mathrm{O}$ desvio padrão de 2,13 confirma a grande variabilidade encontrada nos dados.

Durante a verificação da estacionaridade da série, a análise gráfica inicial dá indícios de que se trata de uma série não estacionária. Ao realizar a análise das funções de autocorrelação (FAC) e autocorrelação parcial (FACP), observou-se que o comportamento das funções realmente segue o padrão de uma série não estacionária e um modelo autorregressivo de ordem 1, ou seja, AR(1), uma vez que apresenta a FAC decaindo rapidamente para zero e a FACP com apenas um lag fora dos limites de controle, como podese observar na Figura 2.

\begin{tabular}{|c|c|c|c|c|c|c|c|}
\hline \multicolumn{2}{|c|}{ Autocorrelation } & Partial Correlation & \multicolumn{2}{|r|}{$\mathrm{AC}$} & PAC & Q-Stat & Prob \\
\hline 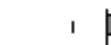 & & 1 & 1 & 0.814 & 0.814 & 41.779 & 0.000 \\
\hline 1 & 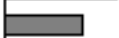 & $\square$ & 2 & 0.580 & -0.244 & 63.369 & 0.000 \\
\hline 1 & $\square$ & $1 \mathrm{~b}$ & 3 & 0.407 & 0.058 & 74.183 & 0.000 \\
\hline I & $\square$ & , G。 & 4 & 0.339 & 0.157 & 81.812 & 0.000 \\
\hline 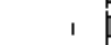 & 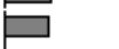 & $1 \longdiv { 1 }$ & 5 & 0.324 & 0.050 & 88.915 & 0.000 \\
\hline I & $\sqsubseteq$ & , G。 & 6 & 0.353 & 0.148 & 97.511 & 0.000 \\
\hline 1 & $巨$ & 1 今 & 7 & 0.391 & 0.093 & 108.22 & 0.000 \\
\hline 1 & 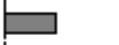 & 101 & 8 & 0.374 & -0.064 & 118.23 & 0.000 \\
\hline 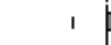 & 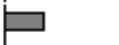 & 1 & 9 & 0.286 & -0.104 & 124.22 & 0.000 \\
\hline 1 & 曰 & ו口 & 10 & 0.243 & 0.175 & 128.61 & 0.000 \\
\hline I & 巨 & 151 & 11 & 0.237 & 0.009 & 132.89 & 0.000 \\
\hline 1 & 巨 & 101 & 12 & 0.225 & -0.074 & 136.82 & 0.000 \\
\hline 1 & פ & 1단. & 13 & 0.143 & -0.194 & 138.44 & 0.000 \\
\hline 1 & 11 & 미 1 & 14 & 0.010 & -0.204 & 138.45 & 0.000 \\
\hline 1 [ & 1 & 111 & 15 & 0.094 & -0.024 & 139.19 & 0.000 \\
\hline
\end{tabular}

Figura 2 - Gráfico das FAC e FACP. Fonte: dados da pesquisa 
Em função disso, identificou-se a necessidade de realizar os testes de raiz unitária Dickey-Fuller e KPSS. O teste Dickey-Fuller Aumentado (DFA) a 1\% de significância mostrou que a série é não estacionária, sendo o valor crítico da estatística $\tau=-3,54$ e o valor calculado $\tau=-2,32$. O teste, portanto, não apresentou evidências estatísticas que refutasse a hipótese $\mathrm{H}_{\mathrm{o}}$ de não estacionariedade. $\mathrm{O}$ teste foi repetido com a série em primeira diferença, sendo o valor crítico da estatística $\tau=-3,54$ e o valor calculado $\tau=-6,36$. Assim, o teste em primeira diferença confirmou que a série tornou-se estacionaria.

Como forma de confirmação do resultado foi realizado também o teste KPSS, a $1 \%$ de significância, cujo valor crítico da estatística $\tau=0,73$ e o valor calculado $\tau=0,13$, rejeitando novamente a hipótese nula e confirmando que a série original é não estacionária.

Na Figura 3, pode-se observar o comportamento da série original (IP) e da série em primeira diferença $\mathrm{D}(\mathrm{IP})$, onde destaca-se na série original a grande variabilidade do índice nos primeiros períodos e certa obtenção de equilíbrio ao final da série. Isto pode ser explicado pelo fato de que a empresa pesquisada entrou em funcionamento no ano de 2009 , período que corresponde também ao início da coleta de dados da série.

A falta de experiência no processo produtivo pode explicar a ocorrência desta grande variabilidade evidenciada, bem como possíveis gaps encontrados nestes primeiros anos, como por exemplo, a falta de pessoal qualificado e ajustes nos equipamentos instalados. No decorrer da série já se observa um maior equilíbrio e consequente estacionarização da série, provavelmente explicada pela obtenção de conhecimento, experiência, qualificação da mãode-obra e ajustes nos equipamentos. Esta estabilização ocorrer nas observações ocorridas cerca de 2 anos e meio depois da instalação da fábrica, e a série em primeiras diferenças já não apresenta os picos tão evidentes e se mostra estacionária, apesar de manter certa variabilidade.

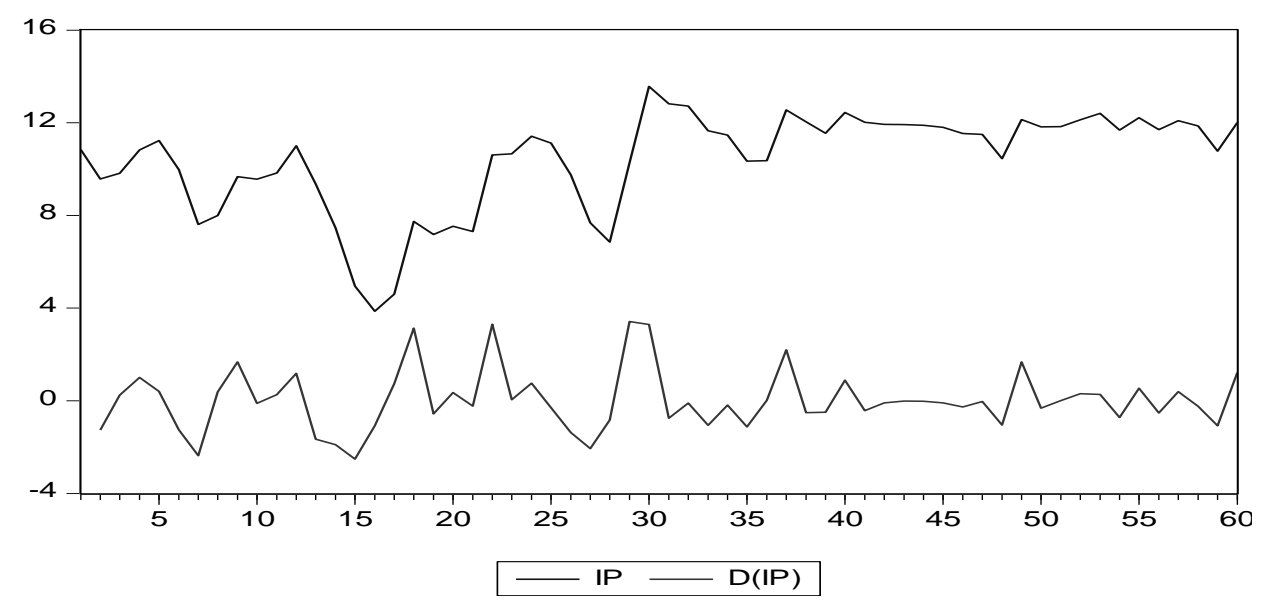

Figura 3 - Gráfico das séries original e diferenciada.

Após tornar a série estacionária por meio de diferenças, o próximo passo é identificar o mecanismo gerador, de modo que os efeitos da autocorrelação fossem captados por meio da escolha de um modelo matemático representativo para a série (SOUZA, LÍRIO e KLIDZIO, $2012^{[30]}$.

Após ser identificado o processo gerador da série pelas funções ACF e PACF, parte-se para a estimação dos parâmetros do modelo, em que encontrou-se quatro modelos concorrentes capazes de explicar o comportamento da série, cujos parâmetros são 
considerados significativos, cujos respectivos coeficientes estimados, níveis de significância dos coeficientes ( $p$-valor) e valores do teste são relacionados na Tabela 1.

Tabela 1 - Modelos concorrentes para a variável de produtividade do leite em pó.

\begin{tabular}{lllll}
\hline Modelo & $\begin{array}{l}\text { Coeficiente } \\
\text { Estimado }\end{array}$ & p-valor & AIC & BIC \\
\hline AR(3) & $-0,319282$ & 0,0173 & 3,319683 & 3,338596 \\
MA(2) & $-0,276549$ & 0,0334 & 3,319380 & 3,354592 \\
MA(3) & $-0,376735$ & 0,0035 & 3,255168 & 3,290380 \\
ARMA(3,1,2) & $-0,609872$ & 0,0169 & 3,283090 & 3,355224 \\
\hline
\end{tabular}

Os valores do AIC e do BIC foram utilizados para determinar o modelo que mais de adequa ao contexto estudado, sendo que isto se dá pela escolha dos menores valores encontrou-se um modelo de médias móveis de ordem 1, com período 3 significante, MA(3), como o melhor modelo, segundo os critérios apresentados.

Este resultado mostra que o processo gerador da série segue um modelo de médias móveis de ordem $1 \mathrm{com}$ influência anterior no período compreendido entre três meses. Isto vai de encontro aos preceitos da produtividade da indústria, pois expressam que, se houver um problema durante o processo de industrialização e o índice se apresentar em baixa naquele respectivo mês, os índices dos próximos também serão negativamente afetados. Da mesma forma, quando há uma melhoria que aumenta o índice de produtividade em um determinado mês, o índice sofrerá influência positiva deste resultado.

Os resíduos proporcionados pelo modelo MA(3) estão apresentados na Figura 4, não são correlacionados, com distribuição aleatória em torno de zero e variância aproximadamente constante, caracterizando-se, portanto, como ruído branco e confirmando a validade do modelo.

\begin{tabular}{|c|c|c|c|c|c|}
\hline Autocorrelation & Partial Correlation & $\mathrm{AC}$ & PAC & Q-Stat & Prob \\
\hline 1 & $1 p 1$ & 10.056 & 0.056 & 0.1932 & \\
\hline । & 만 & $2-0.209$ & -0.210 & 2.8859 & 0.089 \\
\hline 1 & 101 & 30.017 & 0.045 & 2.9046 & 0.234 \\
\hline 1 & 可 1 & $4-0.099$ & -0.140 & 3.4404 & 0.329 \\
\hline 1미 & 1 1 & $5-0.149$ & -0.120 & 4.8403 & 0.304 \\
\hline 11 & 101 & $6-0.029$ & -0.060 & 4.8758 & 0.431 \\
\hline 1 & 101 & $\begin{array}{ll}7 & 0.091\end{array}$ & 0.045 & 5.4486 & 0.488 \\
\hline । 口। & ı 口 & 80.144 & 0.121 & 6.9027 & 0.439 \\
\hline 10 & 1 . & $9-0.069$ & -0.080 & 7.1760 & 0.518 \\
\hline 101 & 1 & $10-0.059$ & -0.020 & 7.4327 & 0.592 \\
\hline 111 & 1 & 110.021 & -0.000 & 7.4667 & 0.681 \\
\hline 口， & , 口 & $12 \quad 0.172$ & 0.226 & 9.7296 & 0.555 \\
\hline 101 & 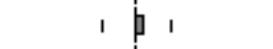 & 130.041 & 0.055 & 9.8641 & 0.628 \\
\hline 1 & 11 & $14-0.099$ & -0.040 & 10.532 & 0.650 \\
\hline 10 & । प्वे । & $15-0.090$ & -0.121 & 11.187 & 0.671 \\
\hline
\end{tabular}

Figura 4 - Resíduos do modelo.

Após esta etapa, partiu-se para a previsão do índice de produtividade através do modelo encontrado, cujo gráfico pode ser observado na Figura 5. Os resultados da previsão para três meses incluíram 59 das 60 observações, obtendo raiz do erro médio quadrado de 
1,211302, média do erro absoluto de 0,954295 e percentual de erro médio absoluto de 10,27. A proporção da variância ficou em 0,008 e da covariância 0,9910 .

De acordo com a previsão realizada, pode-se observar que o comportamento da linha de previsão segue o comportamento da série original, mostrando-se como uma boa previsão para a série estudada.

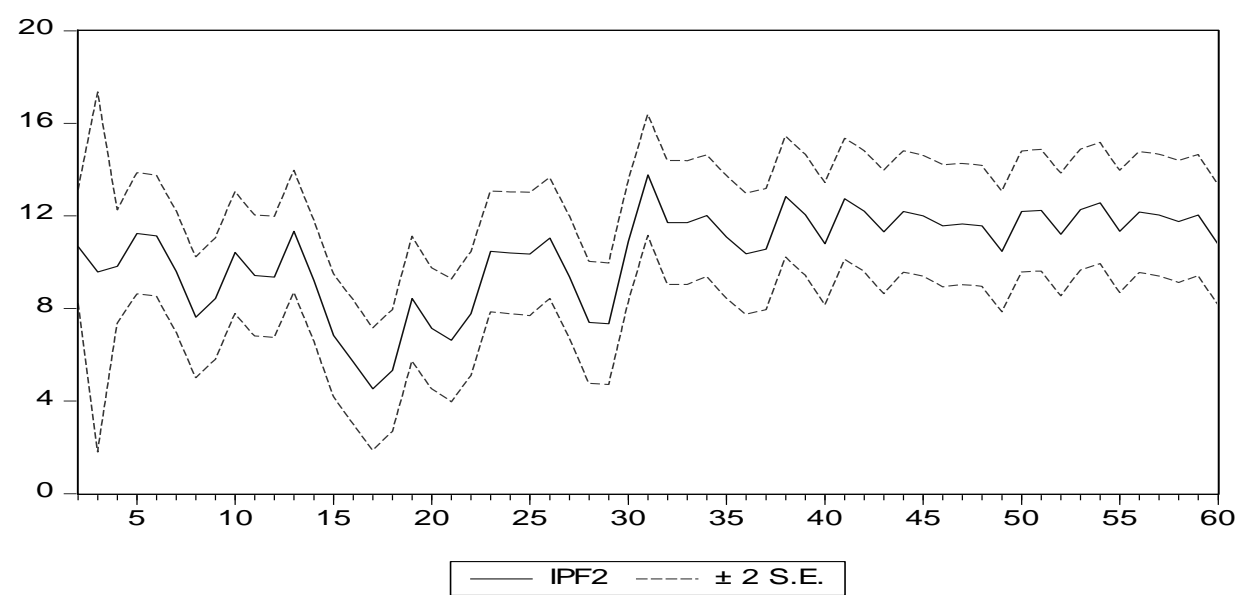

Figura 5 - Previsão do modelo MA(3) ajustado.

A previsão realizada mostra que a serie tende a não ter muitos picos como evidenciado no início do processo de produção, com valores do índice girando entre 9,9 e 9,5, desempenhos próximos da média inicial de 10,39, porém um pouco abaixo da mesma. Para o processo como um todo. A diminuição dos picos muito altos e muito baixos é bastante favorável, por retratar o atingimento de uma maturidade no processo, dando maior precisão para os gestores no momento de planejar ações que visem melhorias.

Esta previsão evidenciou aos gestores que seus esforços na melhoria dos processos internos, por meio do atendimento aos requisitos de normas internacionais, tem gerado frutos positivos e diminuído muitos dos problemas de produção enfrentados nos primeiros anos de funcionamento.

As ferramentas utilizadas nesta pesquisa podem ainda auxiliar na previsão de outros índices da empresa e do setor, visando sempre uma antevisão dos cenários possíveis e melhor alinhamento das estratégias competitivas.

\section{CONSIDERAÇÕES FINAIS}

O presente artigo teve como objetivo realizar previsões do índice de produtividade no curto prazo em uma indústria de laticínios, assim como determinar o processo gerador da série analisada, como forma de buscar subsídios na tomada de decisão de modo a tornar a empresa mais competitiva no mercado nacional e internacional, além de poder corrigir falhas no seu processo e prever o comportamento do seu índice de produtividade.

Observou-se que a série original possui grande variância nos primeiros meses observados, correspondendo ao período dos primeiros anos de funcionamento da indústria pesquisada, o que explica bruscas mudanças no índice de produtividade. Ao longo dos anos correspondentes às últimas observações, já há indícios de uma estabilidade da série, em função da melhoria nos processos da indústria derivados do ganho de experiência no processo. 
Pôde-se concluir que o modelo gerador da série analisada é um modelo AR(3), significando que, se houver um problema durante o processo de industrialização e o índice se apresentar em baixa naquele respectivo mês, os índices dos próximos três meses também serão negativamente afetados. Da mesma forma, quando há uma melhoria que aumenta o índice de produtividade em um determinado mês, o índice sofrerá influência positiva deste resultado.

Conclui-se também que os modelos de classe geral ARIMA são boas ferramentas para a realização de previsão nas empresas, e que a provisão do índice de produtividade da indústria pode colaborar com os gestores na tomada de decisões estratégicas, tanto para antever problemas no seu processo produtivo, quanto para alinhar as vendas com as quantidades de produção que estarão disponíveis nos meses subsequentes.

No caso estudado, a indústria se beneficiou dos resultados com a possibilidade da antevisão do índice de produtividade, fazendo com que gestores pudessem planejar com mais exatidão as ações de melhoria.

As principais limitações da pesquisa estão relacionadas ao número de observações colhidas, que apesar de ser suficiente, poderia obter resultados ainda mais satisfatórios quando baseado em mais observações.

Como a indústria foco do estudo está instalada há apenas cinco anos, não foi possível obter mais observações até o momento, contudo, futuramente o estudo pode ser refeito com mais observações disponíveis.

\section{REFERÊNCIAS BIBLIOGRÁFICAS}

[1] DRUCKER, P.F. Management. New York: Harper Business, 2008.

[2] PORTER, M. Competição. Rio de Janeiro. Elsevier, 2009.

[3] TEMPLAR, R. The rules of management, expanded edition: a definitive code for managerial success. New Jersey: FT Press, 2011.

[4] IRELAND, R.D.; HOSKISSON, R.E.; HITT, M.A. Strategic Management: Concepts and Cases: Competitiveness and Globalization. Boston: South-Western College Publishing, 2014.

[5] MACHADO-DA-SILVA, C. L.; FONSECA, V, S. Competitividade organizacional: uma tentativa de reconstrução analítica. Revista de Administração Contemporânea, v.14, 33-49, 2010.

[6] PAIS, P. S. M.; GOMES, M. S. M.; CORONEL, D. A. Análise da competitividade das exportações brasileiras de minério de ferro de 2000 à 2008. RAM, Revista de Administração Mackenzie, v. 13, n. 4, p. 121-145, 2012.

[7] NEUENFELDT JÚNIOR, A.; SILUK, J. C. M.; SILVEIRA. M. N. Modelo de avaliação de desempenho para os setores do sistema de franquias do Brasil. Cuad. admin. Ser. Organ., v. 26, n. 47, p. 285-307, 2013.

[8] MANÃS, A V.; PACANHAN, M. N. Strategic Alliances and Associativism Networks as a Source of Competitiveness Advantage in the Retailers Market of Construction. Review of Business Management, v. 6, n. 14, p. 19-33, 2004.

[9] ZAGO, C.A.; RIGONI, J.; ABREU, L.F.; RODRIGUEZ, C.M.T. Perspectivas metodológicas de avaliação de desempenho organizacional: aplicabilidade na logística. Revista Eletrônica Sistemas \& Gestão. v. 3, n. 3, p. 178-195, 2008. 
[10] FONTENELE, R. E. S. Empreendedorismo, competitividade e crescimento econômico: evidências empíricas. Revista de Administração Contemporânea, v.14, n.6, 2010.

[11] MOREIRA, A C.; MOUTINHO, V. F.; PEREIRA, J. C. Evaluación de una Estrategia Colaborativa: un estudio de caso en el sector del vino de Oporto. Review of Business Management, v. 15, n. 47, p. 221-240, 2013.

[12] CARLINI JUNIOR, R. J.; VITAL, T. W. The use of Benchmarketing in the elaboration of Strategic Planning: an important tool for the maximization of the organizational competitiveness. Review of Business Management, v. 6, n. 14, p. 60-66, 2004.

[13] NEELY, A. The evolution of performance measurement research: developments in the last decade and a research agenda for the next, International Journal of Operations \& Production Management, v. 25, n. 12, p. 1264-1277, 2005.

[14] BITITCI, U. S.; MENDIBIL, K.; MARTINEZ, V.; ALBORES, P. Measuring and managing performance in extended enterprises. International Journal of Operations \& Production Management, v. 25 n. 4, p. 333-53, 2005.

[15] SLACK, SLACK, N.; CHAMBERS, S.; JOHNSTON, R. Operations strategy. Upper Saddle River, New Jersey: Prentice Hall. 2010.

[16] HUBBARD, G.; RICE, J.; BEAMISH, P. Strategic Management: Thinking, Analysis and Action. São Paulo: Pearson Education, 2011.

[17] ABIA. Associação Brasileira das Indústrias da Alimentação. O setor em números. 2012. Disponível em: < http://www.abia.org.br/vs/inicio.aspx>. Acesso em: 14/10/2014

[18] IBGE. A cadeia do leite. 2011. Disponível em: http://saladeimprensa.ibge.gov.br/noticias. Acesso em: 12/02/2014.

[19] MILKPOINT. Produção de leite no Brasil. 2012. Disponível em: http://www.milkpoint.com.br/estatisticas/Producao_Mundial.htm. Acesso em: 07/02/2014.

[20] LÍRIO, G. W.; SOUZA, A M.; DA SILVA, W. V. Análise e previsão da série recebimento e produção de leite da usina escola de laticínios da Universidade Federal de Santa Maria. Revista Ciência \& Natura, Santa Maria, v. 25, p. 23-40, 2003.

[21] GUJARATI, D. N. Econometria básica. São Paulo: Makron Books, 2000.

[22] ANDRADE, P. C, R. estudo da série da taxa de desemprego na região metropolitana de Recife. Revista da Universidade Vale do Rio Verde, v. 9, n. 1, p. 03-18, 2011.

[23] BOX, G. E. P.; JENKINS, G. M. Time Series Analysis: Forecasting and Control. San Francisco: Holden-Day, 1970.

[24] SOUZA, A. M.; SOUZA, F. M.; FERREIRA, N.; MENEZES, R. Eletrical energy supply for Rio Grande do Sul, Brazil, using forecast combination of weighted eigenvalues. GEPROS, Gestão da produção, operações e sistemas. v. 6, n. 3, p. 23-39, 2011.

[25] STADLHOFER, G. Corporate to core business competitiveness at global pharmaceutical enterprise. Journal of Corporate Real Estate, v. 12, n. 2, p.96-116, 2010.

[26] JOSHI, D.; NEPAL, B.; RATHORE, A. P. S.; SHARMA, D. On supply chain competitiveness of Indian automotive component manufacturing industry. International Journal Economics, v. 143, p. 151-161, 2013. 
[27] MORETTIN, P. A.; TOLOI, C. M. C. Previsão de séries temporais. São Paulo: Atual, 2004.

[28] MACKINNON, J. G. Numerical distribution functions for unit root and cointegration tests. Journal of Applied Econometrics. v. 11, p. 601-618. 1996.

[29] KWIATKOWSKI, D.; PHILLIPS, P.; SCHMIDT, P. SHIN, Y. Testing the null hypothesis of stacionarity against the alternative of a unit root. Journal of Econometrics. v. 54, p. 159-178, 1992.

[30] SOUZA, A. M.; LÍRIO, G. W.; KLIDZIO, R. Análise da estabilidade da produção de leite no município de Santo Ângelo - RS. Revista Ciência e Natura. v. 34, p. 7-26, 2012. 